\title{
Self-similar Sets as Tarski's Fixed Points
}

By

\author{
Susumu HAYASHI*
}

J. E. Hutchinson [4] and M. Hata [2] investigated some self-similar fractals as the unique non-empty compact solution $X$ of the equation

$$
\text { (A) } \quad K=F_{1}(X) \cup \cdots \cup F_{n}(X) \text {, }
$$

where each $F_{i}$ is a contraction of $\mathbb{R}^{p}$. They showed the solution is the unique fixed point of a contraction of the complete metric space of non-empty compact sets of $\boldsymbol{R}^{p}$. In this note, we will show that the solution may be thought as Tarski's fixed point of a continuous function of a complete ordered set.

Definition 1. A complete partial order, CPO in short, is a partial order ( $D$, ㄷ) such that (i) $D$ has the bottom element $\perp$, and (ii) every directed set $X$ has the sup $\sqcup X$ (cf. [1]). A function of $\mathbb{C P O}$ is said continuous, if and only if it preserves the sup of every directed set, i. e. $\sqcup f(X)=f(\sqcup X)$. Note that continuous functions preserve the order.

Theorem 1 (Tarski's fixed point theorem). If $f$ is a continuous endomorphism of a $\mathbf{C P O}$, then $\cup f^{n}(\perp)$ is the least fixed point of $f$.

Let $S$ be a compact Hausdorff space. Set

$$
\begin{aligned}
& D=\{K \subseteq S \mid K \neq \phi, K \text { is closed }\}, \\
& a \sqsubseteq b \text { if and only if } b \leqq a(a, b \in D) .
\end{aligned}
$$

Then $(D$, 드) is a CPO with the bottom $S$, since every directed set in $D$ has a non-empty intersection. Note that a topological space is compact if and only if its partially ordered set of non-empty closed sets $(D$, 드) is a $\mathbb{C P O}$. Any subset of $D$, say $X$, has the inf $\sqcap X$, which is the closure of $\cup X$. Besides, $a_{1} \sqcap \cdots \sqcap a_{n}$ is a continuous $n$-ary function, i. e. continuous with respect to each argument $a_{i}$. We will call this kind of $\mathbf{C P O}$ a spatial $\mathbf{C P O}$.

On the other hand, $D$ is a compact Hausdorff space with the finite topology [6], which is generated by bases of the form

Received May 30, 1985.

* Research Institute for Mathematical Sciences, Kyoto University, Kyoto 606, Japan. 


$$
\left\{K \mid K \leqq \cup U_{\imath}, \forall i U_{i} \cap K \neq \phi\right\},
$$

with open sets $U_{1}, \cdots, U_{n}$ of $S$. So we have two kinds of continuity of the functions of $D$. To distinguish them, we introduce the following terminologies.

Definition 2. An endomorphism $f$ of $D$ is said $T$-continuous, if and only if $f$ is continuous in the sense of the finite topology. On the other hand, $f$ is said O-continuous, if and only if $f$ is continuous in the sense of $\mathbf{C P O}$.

Lemma 1. Every directed set $X$ in $D$ converges to $\sqcup X$ in the sense of the finite topology.

Proof. Let $X$ be a directed set in $D$. Then $X$ defines a net in the topological space $D$ of the finite topology. Since the space $S$ is compact, this net converges to the point $\sqcup X$.

Corollary 1. If $f: D \rightarrow D$ is order-preserving and T-continuous, then it is $O$ continuous.

Remark 1. Note that the converse does not hold. The following function is $O$-continuous, but it is not $T$-continuous, when $a$ is an accumulation point:

$$
f(X)= \begin{cases}S & a \in X \\ \{a\} & \text { Otherwise. }\end{cases}
$$

We will give another example, later.

Assume $F$ is a continuous endomorphism of a compact Hausdorff space $S$. Let $F^{*}$ be the endomorphism of $D$ defined by $F^{*}(X)=F(X)$. Then $F^{*}$ is $T$ continuous.

Theorem 2. Let $F_{1}, \cdots, F_{2}$ be continuous endomorphisms of $S$. Then the following equation has the greatest solution.

$$
X=F_{1}(X) \cup \cdots \cup F_{n}(X) \quad(X \cong S) .
$$

Besides, the solution $X$ is non-empty and compact.

Proof. Since $S$ is compact, $F_{\imath}(\bar{X})=\overline{F_{\imath}(X)}$. So it is sufficient to find the least fixed point of the following continuous endomorphism of $D$ :

$$
F(x)=F_{1}^{*}(x) \sqcap \cdots \sqcap F_{n}^{*}(x) .
$$

By Theorem 1, such a fixed point exists.

By virtue of the above general argument, we can realize self-similar sets of [2], [4] as Tarski's fixed points. Let $F_{1}, \cdots, F_{n}$ be contractions of $\boldsymbol{R}^{p}$. Let 
the Lipschitz constant $\operatorname{Lip}\left(F_{\imath}\right)$ be $\lambda_{\imath}, p_{\imath}$ be the fixed point of $F_{\imath}$, and $d_{\imath}$ be $d\left(a_{1}, a_{\imath}\right)$. Since $0 \leqq \lambda_{2}<1$, there exists $\varepsilon>0$ such that

$$
\lambda_{2}\left(\varepsilon+d_{2}\right)+d_{2}<\varepsilon \quad(i=1, \cdots, n) .
$$

Let $S$ be the closed ball $B\left(a_{1}, \varepsilon\right)$. Then $F_{l}(S) \leqq S$. (This argument is due to [10].) Hence, the self-similar sets of [2], [4], each of which is the unique solution of the equation of the form (A), are Tarski's fixed points in the CPO of non-empty compact sets of $S$. Let $F(X)$ be the right-hand side of the equation (A). Note that the solution is the intersection of the decreasing sequence of sets

$$
S \supseteqq F(S) \supseteqq F^{2}(S) \supseteqq \cdots
$$

In another word, the solution is the limit set approximated by these from outside. Since any approximation does not shadow the other approximations, we can see the dynamic process creating a self-similar set in a static figure. We disply such figures in figuer 1-4. All of those are fractals of M. Hata [2] except the Koch curve (fig. 1). Each of them is the solution of the equation of the form (A).

The contractions of the equations of Hata's fractals are as follows:

figure 2. $\quad F_{01}(z)=(1 / 2+\sqrt{3} / 6 i) \bar{z}, \quad F_{1}(z)=(2 \bar{z}+1) / 3$,

figure 3. $\quad F_{0}(z)=(1+i) z / 2, \quad F_{1}(z)=((3-i) z+2+i) / 5$,

figure 4. $\quad F_{0}(z)=2^{-1 / 3} e^{(\pi / 3)} z, \quad F_{1}(z)=(z+1) / 2$,

where $z$ is a complex number. The numbers under each figure indicate the displayed approximations. The first approximation is the ball $B(0,1)$ except the fractal of figure 3 , whose first approximation is $B(0,1.2)$. Those figures were drawn by programs written by Takashi Sakuragawa.

Hata [2] pointed out that the following equation has the greatest non-empty compact solution, although it is not of the form of (A).

$$
X=f_{1}\left(X \cap A_{1}\right) \cup f_{2}\left(X \cap A_{2}\right) .
$$

where $A_{1}=[0, a], \quad A_{1}=[a, 1] . \quad f_{1}(s)=1+b(s-a)$, and $f_{2}(s)=b(s-a)$ with the conditions $0<a<1,0<b<1$. Let $g(x)$ be the right hand side of (B). Then $g(x)$ is not $T$-continuous, but it is $O$-continuous. So the existence of the greatest solution follows from Theorem 1. This gives an example of the lattice theoretic approach which cannot be captured by the metric space approach. We gave an $O$-continuous function which is not $T$-continuous in Remark 1. But this example is much more interesting than the example of Remark 1 , since the above equation originates in a study of a discreate dynamical system (cf. [2]). Note that the specific definition of $f_{1}, f_{2}$ of $(\mathrm{B})$ is not relevant to the existence of the greatest solution. In fact, we can show the following theorem (cf. Theorem 3.6 of $[2])$. 


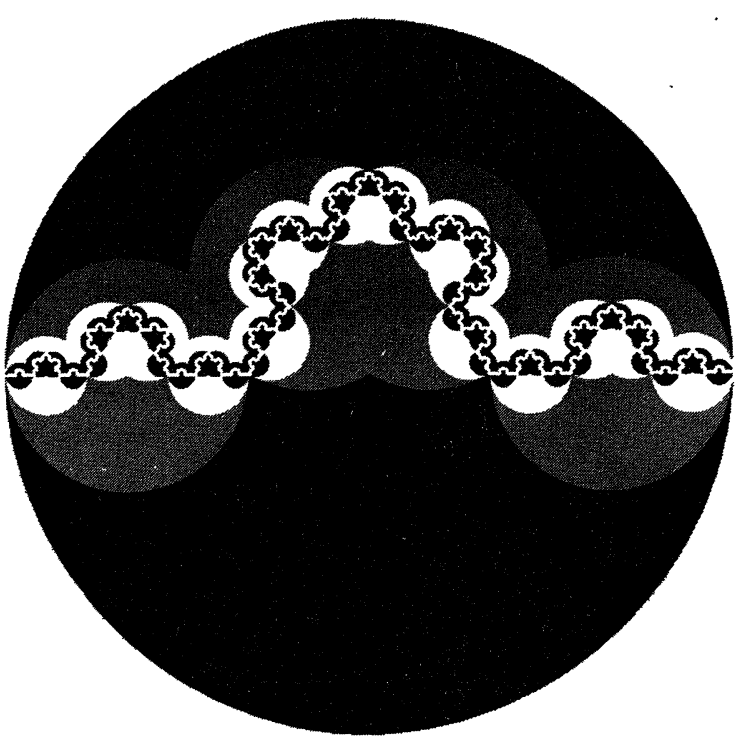

Figure 1. Approximations $=1,2,3,4,6$

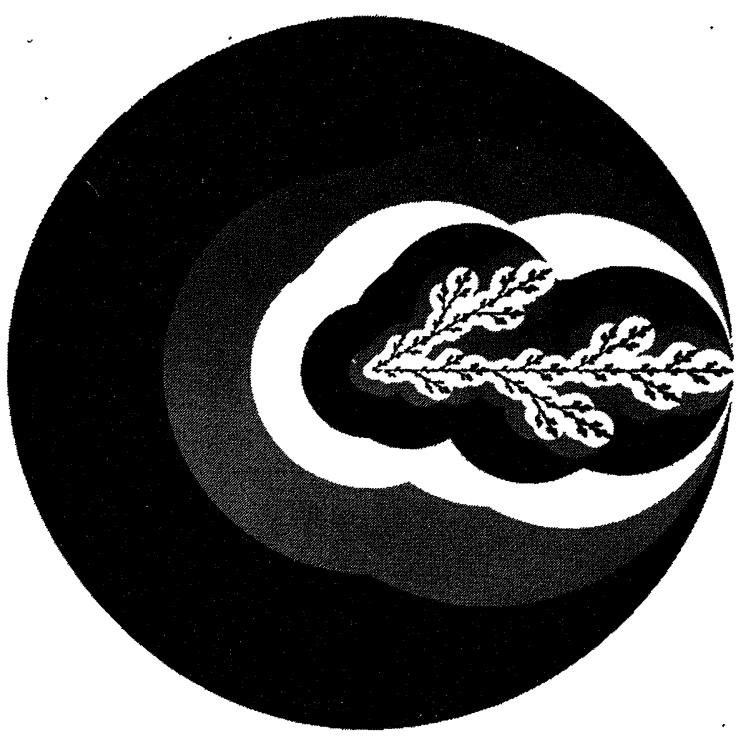

Figure 2. Approximations $=1,2,3,4,6,8,13$

Theorem 3. Let $S$ be a compact Hausdorff space, and let $A_{1}, \cdots, A_{n}$ be a nonempty compact covering of $S$. Assume $F_{\imath}$ is a continuous function from $A_{i}$ to $S$ for each $i=1, \cdots, n$. Then the equation

$$
X=F_{1}\left(X \cap \dot{A_{1}}\right) \cup \cdots \cup F_{n}\left(X \cap A_{n}\right)
$$

has the greatest non-empty compact solution. 


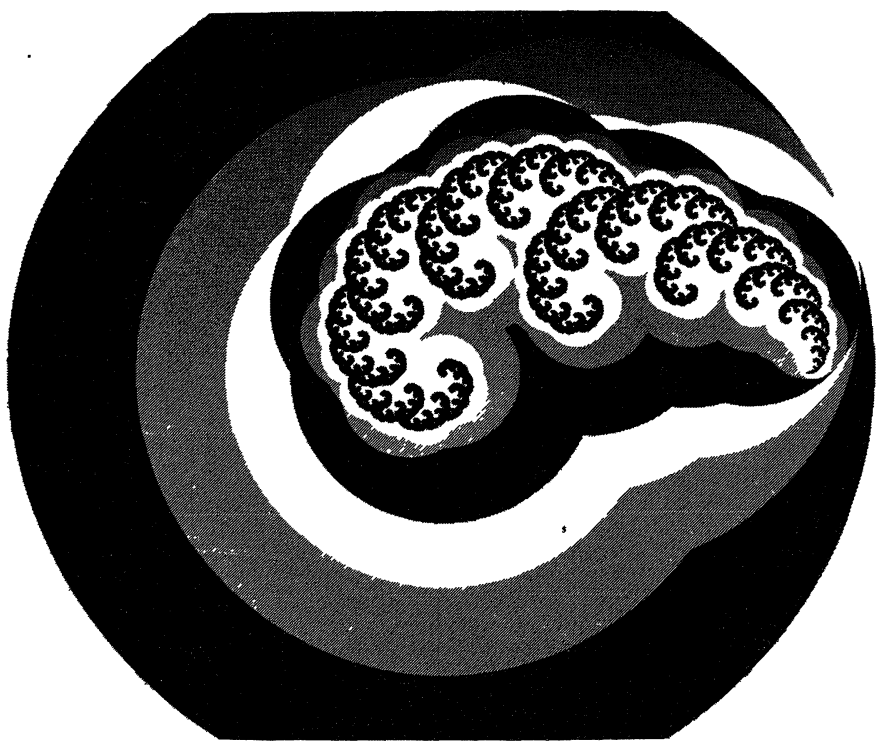

Figure 13. Approximations $=1,2,3,4,6,8,15$

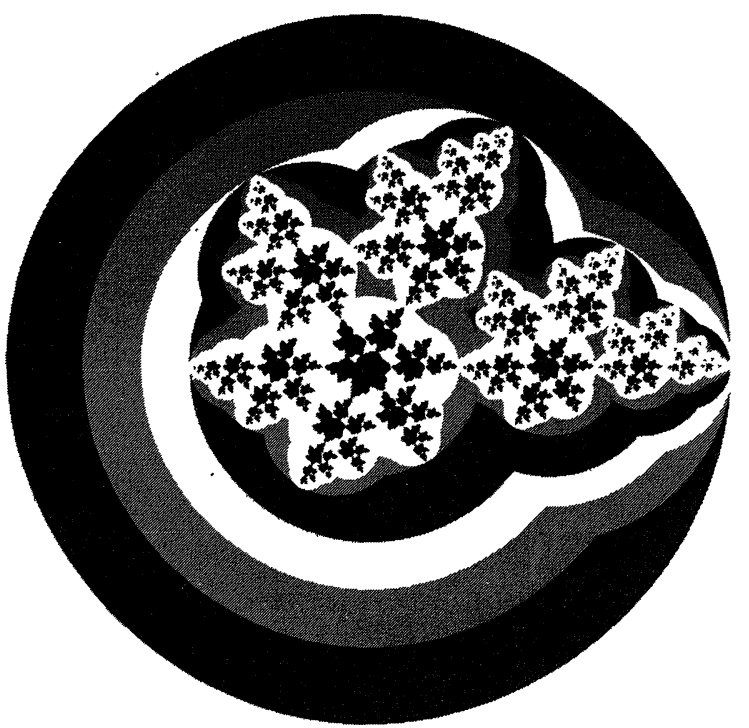

Figuer 4. Approximations $=1,2,3,4,6,8,15$

Another example of a fractal as Tarski's fixed point is the set $J(\alpha)$ associated with the mapping $\phi_{a}(z)=z^{n}-\alpha(n=2,3 \cdots)$, where $z$ and $\alpha$ are complex numbers (cf. Chapter 19 of [5]). The fractal set $J(\alpha)$ is defined as the set of points $z$ such that $\lim z_{n} \neq \infty$, where $z_{0}=z$ and $z_{n+1}=\phi_{a}\left(z_{n}\right)$. It is easily checked that $J(\alpha)$ is represented as $\left\lfloor f^{n}(\perp)\right.$, where $\perp$ is the closed ball $B(0, \alpha+1)$ and $f$ is the $O$-continuous function defined as $f(X)=\phi_{a}^{-1}(X)$. Note that $J(\alpha)$ is also an example 
of the fixed point of Theorem 3. Sakuragawa noted the following facts. Define $\perp$ and $F$ by

$$
\begin{aligned}
& \perp=\left\{(u, v)|| u|\leqq| v^{\prime}|+1,| v \mid \leqq 2\right\}, \\
& F\left(u, v^{\prime}\right)=\left(u^{2}+v^{\prime}, v^{\prime}\right),
\end{aligned}
$$

where $u$ and $v$ are complex numbers. Let $M$ be $\sqcup f^{n}(\perp)$, where $f=F^{-1}$. Then the section $\{u \mid(u, \alpha) \in M\}$ is $J(\alpha)(\alpha \leqq 2)$, the section $\{\alpha \mid(0, \alpha) \in M\}$ is Mandelbrot set (the bottom of Plate 188 of [5]), and $M \cap C \times[-1 / 4,2]$ is Mandelbrot's "draped sculpture" of Plate 187 of [5].

Soto-Andrade \& Varela [9] suggested a relation between Tarski's fixed points and fractals. But. their arguments are unclear and unsatisfactory. Besides, our CPO seems to be much more universal and natural than the CPO suggested by them. They pointed out that Koch curve could be derived by a diagonal argument, if their space of rectangles was embedded in a reflexive domain. This holds in our case, since any CPO is a retraction of a reflexive CPO by Scott's $D_{\infty}$ construction (cf. [1], [7], [8]). Hence any self-similar sets of [2], [4] can be derived by Curry's paradoxical combinator (cf. [1], [7]). This observation may appeal to some readers, although we do not know any specific implications from it.

So far we have not considered the uniqueness of the solution of (A), although Hutchinson [4] and Hata [2], [3] have investigated it. In their setting, each $F_{\imath}$ is a contraction of a metric space, so the uniqueness is an easy consequence. On the other hand, the spaces in our setting are compact Hausdorff space, which are not always metorizable, and each $F_{\imath}$ is any continuous functions. So we need extra conditions for the uniqueness of solutions. We will give such a condition, generalizing Hutchinson's argument (cf. $[4,3.1]$ ). Let $[n]$ be the set $\{1, \cdots, n\}$, let $W$ be the set of finite sequences from $[n]$, and $C$ be the Cantor space $[n]^{N}$. Assume $(D, \sqsubseteq)$ is a CPO and $f_{1}, \cdots, f_{n}$ are $O$-continuous endomorphisms of $D$. Set $f(x)=f_{1}(x) \sqcap \cdots \sqcap f_{n}(x)$. Let $k$ be any fixed point of $f$. Then set

$$
k_{\imath_{1} \cdots \imath_{n}}=f_{\imath_{1}} \circ \cdots \circ f_{\imath_{n}}(k) \text {. }
$$

Then $k=\Pi\left\{k_{w}|w \in W| w \mid,=n\right\}$ for each $n \in N$. Hence we can set

$$
k_{a}=\sqcup k_{\bar{\alpha}(n)} \quad(\alpha \in C),
$$

where $\bar{\alpha}(n)$ is the finite sequence $\alpha(1), \cdots, \alpha(n)$. Since $\perp$ is the least element, we can define $\perp_{a}$, similarly. If the conditions

$$
\begin{aligned}
& \perp_{\alpha} \text { is maximal, } \\
& k=\sqcap k_{a},
\end{aligned}
$$

hold, then $k$ is uniquely determined as $\Pi \perp_{\alpha}$. Note that (C.2) holds for any spatial CPO by a purely set-theoretic reason. On the other hand, if $f_{1}, \cdots, f_{u}$ are induced from contractions of a metric space, then $\perp_{a}$ consists solely a point, 
for the diameter of $\perp_{\bar{\alpha}(n)}$ decreases. Then the condition (C.1) holds, since a point is a maximal element in a spatial $\mathbb{C P O}$. This generalizes the uniqueness result in $[2],[4]$.

Remark 2. Hata [3] considers inhomogeneous equation of the form $x=F(x) \cup v$ and showed the solution is unique if the homogeneous equation $x=F(x)$ has a unique solution, under a condition on $F$. The result remains valid in our setting. The equation $x=f(x) \sqcap v$ in a spatial CPO has a unique solution, if the equation $x=f(x)$ has a unique solution, whenever $f$ is $O$-continuous. Note that Hata's solution of the inhomogeneous equation, $R_{F}=$ the closure of $\cup F^{n}(v)$, is the greatest solution $\sqcap F^{n}(v)$ from our viewpoint.

Remark 3. The solution $K$ of (A) is also characterized as the closure of the set of fixed points of compositions of contractions $F_{i_{1}} \circ \ldots \circ F_{i_{n}}$ (cf. [4], [10]). This holds for any continuous endomorphisms $F_{1}, \cdots, F_{n}$ of a compact Hausdorff space with the property (C.1), since the mapping $\alpha \mapsto \perp_{\alpha}$ is continuous in the sense of the finite topology.

\section{Acknowledgement}

I would like to thank M. Hata, T. Sakuragawa and Prof. H. Barendregt for valuable discussions. I appreciate Sakuragawa's contribution drawing the figures of fractals. I also would like to thank Prof. M. Yamaguti and Prof. R. Nakajima who directed my interest to the subject.

\section{References}

[1] H.P. Barendregt, The Lambda Calculus, Its Syntax and Semantics, North-Holland, Amsterdam, 1981.

[2] M. Hata, On the Structure of Self-similar Sets, to appear in Japan J. Appl. Math..

[3] M. Hata, On Some Properties of Set-dynamical Systems, to appear in Proc. Japan Acad..

[4] J.E. Hutchinson, Fractals and Self-similarity, Indiana Univ. Math. J., 30, pp. 713-747, 1981.

[5] B.B. Mandelbrot, The Fractal Geometry of Nature, Freeman, San Francisco, 1982.

[6] E. Michael, Topologies on Spaces of Subsets, Trans. Amer. Math. Soc., 71, pp. $152-182,1951$.

[7] D.S. Scott, Continuous Lattices, in Toposes, Algebraic Geometry and Logic, F. W. Lawvere (ed.), LNM 274, Springer Verlag, Berlin, 1972.

[8] M.B. Smyth, G.D. Plotkin, The Category-theoretic Solution of Recursive Domain Equations, Internal Report of Department of Computer Science CSR-102-82, University of Edinburgh, Feb. 1982.

[9] J. Soto-Andrade, F. J. Varela, Self-Reference and Fixed Points: A Discussion and an Extension of Lawvere's Theorem, Acta Applicandae Mathematicae, 2, pp. 1-19, 1984.

[10] R.F. Williams, Composition of contractions, Bol. Soc. Brasil. Math. 2, pp. 55-59, 
1971.

P.S. Prof. Dana Scott pointed out that our COP was well known to the authors of "A COMPENDIUM OF CONTINUOUS LATTICES, G. Gierz. et. al., Springer-Verlag, Berlin, 1980". They consider the open-set lattice of a locally compact space, say $O(X)$ for a locally compact space $X$, instead of closed sets (see Chapter I, 1.7, (5) in the Compendium). Our CPO is equivalent to $O(X)-\{X\}$, and Proposition 1.4 of Chapter I says that $O(X)-\{X\}$ is a CPO. I would like to thank Prof. Dana Scott for this and other valuable comments on the paper. I also would like to thank Prof. Gordon Plotkin for pointing our a terminological error. 